\title{
Make a Good Report on Health Management of Occupational Groups
}

\author{
He Aimei Liu Shiquan Li Jisheng \\ Occupational Diseases Prevention and Treatment Institute of Xiangyang of Hubei Province 4410241
}

Received: 20 December 2019 Accepted: 25 December 2019 Published: 31 March 2020

Cite this Article He Aimei, Liu Shiquan, Li Jisheng. Make a Good Report on Health Management of Occupational Groups [J].Medical Research,2020.2(1): 26-31, http://dx.doi.org/10.6913/MRHK.20200 3_2(1).0006

Copyright (C) 2020 Creative Publishing Co., Limited. All rights reserved. Email: kycbshk@gmail.com.

\section{ABSTRACT}

Target Dynamic intervention and management must be implemented among occupational groups for efficient increase of the awareness to manage occupational health.

Methods The relationship among checkup institutions, employers, and occupational health is integrated into the process before, during, and after the checkup and relevant linkage module of the work is designed.

Results The operative module for communication among internal design institutions, employers, and occupational health is designed after years of practice, which plays an important role in dynamic management of occupational health in its practical operation. Dynamic management on occupational health via linkage module realizes both higher working efficiency and social effects.

Conclusion Dynamic management of occupational health is good for common protection on occupational health.

Key Words occupational health; management; practice

The Technical Specification for Occupational Health Monitoring plays a crucial instructive role for China to improve its service level of occupational health examination and realize its smooth operation. However, there are still inharmonious factors which affect the implementation of dynamic management of occupational health before, during, and after the checkup. Some of there factors come from checkup institutions, some employers, and others occupational groups. During the dynamic management of occupational health which lasts several years, positive exploration and improvement were made to implement dynamic management and intervention of occupational health, reducing potential negative factors and chronic diseases caused by bad lifestyles ranging from preventing the occurrence of occupational diseases and dynamic management on individual health. Here is the summary on how to implement dynamic management on individual health in occupational groups:

\section{I.Occupational Health Needs Agreement in Implementing Dynamic Management}

Key Points of Agreement in Dynamic Management 
1. The staff involving checkup in checkup institutions for occupational health must know the names of occupational hazardous factors existing in the employers, figure out whether a difference is an individual one or a consequent one from the checkup results, and find the problems from the checkup results of occupational groups for early prevention of occupational diseases.

2. If checkup institutions of occupational health and the employers want to add extra items on the original obligatory items, they must explain the pros and cons in order to decide whether the item must be examined. The employees' information offered by the employer must be true and comprehensive to meet the demands of occupational health checkup.

3. The occupational groups must have adequate communication with the physician about their subject expectations and object conditions during checkup as professional checkup contains legal compulsory.

4. A service mode with agreement with the employer on the basis of agreement for the implementation of dynamic management of occupational health.

\section{Here Are the Contents Needed in the Report on Health Management of Occupational Groups}

\section{There must be clear explanation on abnormal occupational indicators and other abnormal ones}

First, everybody draws conclusion according to the regulation that the majority of chief physicians in the charge of occupational health examination analyze and comb the results of checkup according to theories, found their relativity, and put forward disposals related to the occupation in accordance with regulated requirements. This is only the judge from systemic analysis on the employees' checkup results which however fails to reflect the physical fitness and health, living habits, operating habits, adaptation of social factors, and places of operation of the occupational groups.

It is inevitable for the chief physicians to have incomprehensive and inaccurate judge on the checkup results as they lack expertise in occupational health and communication with occupational groups except that they can adopt their abundant clinical medicine expertise and experience. Therefore, a personalized report module must be designed for the occupational groups for accurate, objective, and prompt reports.

\section{The Transmission Functions of Papery Media with Expertise in Hazardous Factors of Occupational Diseases}

The report must filter the content of propaganda on hazardous factors of occupational diseases according to the places of operation as the transmission on hazardous factors of occupational diseases is a routine work. All kinds of recyclable linkage modules with occupational health knowledge must be designed to present its contents to relevant personnel of all levels promptly and send the correct knowledge and information on occupational health to the occupational groups to shift their awareness of managing occupational health. Such papery media plays an important role in continuous transmission of expertise in preventing occupational diseases to the occupational health.

\section{Summary and Sequence of Report on Health Management of Occupational Groups}

Not all the contraindications and suspected occupational diseases can be judged from the checkup results for one cause or another, as some checkup results presents the abnormal 
indicators tightly related to the occupation. More scientific summary and sequence of the report will benefit both occupational groups and employers, which will be demonstrated as follows. A. Conclusion of Report (1) Hazardous factors of occupational diseases:Including name, occupational sorts, and measurement of concentration and intensity of hazardous factors of occupational diseases in the occupational places. (2) Abnormal indicators highly related to the occupation:Such as $\mathrm{Pb}-\mathrm{B}$ increase, and benzene-induced leukopenia.(3) Definite diagnosis of chronic diseases. (4) Other abnormal results:Such as benign lesion, degenerative change, hepatic adipose infiltration, cholecystolithiasis, and etc.

B. Sequence of medical departments:internal medicine, surgery, ophthalmology and otorhinolaryngology, function departments, and gynecology.

C. Evaluation on risk of hazardous factors of occupational diseases Evaluation on occupational health of occupational groups can be made according to the checkup results which can add a tip to remind the occupational groups to pay attention to their health. When it comes to other diseases, suggestions on health, prompt re-examination, tracking and watch, further examination, and prompt treatment must be given according to the requirements of ICD-10.

\section{Report on Health Management of Occupational Groups Must Have the Following Features}

Only with the following features can there be a qualified report on health management of occupational groups.

1. Comprehensive contents:The conclusion of occupational health examination must reflect the employees' physical conditions comprehensively, objectively, and truly, especially no misjudge on their occupational contraindications and suspected occupational diseases, which constitutes the fundamental requirements of report on health management of occupational groups.

2. Clear logic The conclusion of report on health management of occupational groups adequately reflects the chief physician's abilities to adopt expertise in occupational health and clinical thinking, in which they must find the relationship between the results and the occupation from the checkup results, make judges, find all the abnormal indicators related to the occupation without missing a single one, and make the employees clear about the current primary and secondary problems affecting their health.

3. Clear levels The sequence of chief physician's report on health management of occupational groups by the chief physician and on other diseases must be unified and sequent for more normality and logic through national academic conferences where the experts in the community can reach a fundamental agreement.

4. Regulation of Terminology The terminology of checkup results by the chief physician and related to the occupation must be written in accordance with the regulation while that of other diseases must be written in accordance with ICD-10 for further regulation of occupational health examination.

\section{The Following Principals Must be Observed in Report on Health Management of Occupational Groups}

1. Evidence-based principle The chief physician explaining the report on health management of occupational groups must have a title higher than a middle-level, with not only abundant clinical experience but also fundamental expertise in prevention and treatment of occupational diseases and attention to the updating of relevant theoretic knowledge. The chief physicians and physicians of all departments must be organized often to learn and master the latest 
diagnosis and treatment guideline and experts' agreement on diseases tightly related to checkup issued both home and abroad. The average level and quality of report on health management of occupational groups must be improved as they examine the diseases related to an occupation according to Technical Specification for Occupational Health Monitoring.

2. Principle of personalizationOne-to-one suggestion and instruction must be made in report on health management of occupational groups according to the physical fitness and health of occupational groups and status of operating places, as the conclusion generated merely by the software and system lack pertinence so that they can not be copied completely in accordance to the regulation. The meaning of examination on abnormal indicators must be detailed, true, and accurate, increasing its consistency, systematicness, and pertinence.

3. Principle of consistency An agreement must be reached among the suggestions given by all the physicians involving the checkup, without any conflicts. The chief physician must measure the pros and cons of occupational health and their operating places to make scientific judges. If there is any conflict in the disposals given by physicians involving the checkup, the occupational groups will not know what to do.

4. Principle of dynamics After several checkups on occupational groups, a dynamic comparison must be made among the checkup results and key indicators related to the occupation, with a dynamic trend graph given. It there is inconsistency between two points or between results and reality, the reason why they ascend or descend must be clear and judges must be made with results of more than one test. Judges must be clear with instructions given to the employees. The regulation is the principle so conception of dynamic management forms the golden standard of regulation.

\section{Report on Health Management of Occupational Groups Not Only Explains Occupational Contradictions}

Results and judges in report on health management of occupational groups is a kind of instructive explanation with legal policies. There will be many problems when explaining occupational contraindications in real process of the work. The employees' economic interests and individual will must be taken into proper consideration when detecting their occupational contraindications on the basis of protecting the occupational groups' health. Conflicts between occupational groups and employers must be prevented and it must be eliminated that employers get economic benefits at the expense of occupational health in regardless of the regulations of Law of Prevention and Treatment of Occupational Diseases. We must carry on three regulations:1 Explain the results and judges of other diseases with unified terminology; (2) If we need to filter and examine the occupational contraindications, we must have dynamic judges in accordance with the Technical Specification for Occupational Health Monitoring according to the concentration of hazardous factors in the places of operation, sorts of occupation, operating positions, time and space, and anamnesis. If decision can not be made by one test, we must head to the employers' places to negotiate with employers and employees for conclusions considering the concrete conditions.(3) Conclusions on suspected occupational diseases must be drawn after strict filtering and examination according to steps (1) and (2), without mental load added to the objected occupational groups.

\section{The Importance of Continuous and Real Records of Factors Related to Occupational Diseases}


The history of exposure of hazardous factors of occupational diseases must be filled carefully in every checkup to ensure the quality of report on health management of occupational groups. There will be lack of objectiveness without real original information so that the accuracy of disposals will be hard to ensure. Especially the disposals on abnormal indicators related to the occupation must be sent after the clinical physician and the occupational health physician reach an agreement under an established review and audit system of chief physicians' report of checkup. The conclusions which are hard to define must be reported with concrete evidence through re-examination and panel discussion and etc according the working guideline of occupational health and prevention and treatment of occupational diseases.

\section{It Is of the Same Importance to Explain Other Abnormal Indicators}

At present, the terminology to explain other abnormal results is not unified. Therefore, the terminology of reports must be unified according to the requirements of the Interim Provisions on the Administration of Health Examination issued by the National Health Department in 2009 and Guidelines for Quality Control of Health Examinations in 2016 The Report on Health Management of Occupational Groups must contain the following: Evaluation on occupational health, disposals of results of occupational health checkup, and suggestions on occupational health; Disposals, health evaluation, and health suggestions in terms of results of other diseases. Primary and secondary contents and responsibilities must be made clear. Besides careful implementation of preventing and treating occupational diseases, explaining results of other abnormal indicators also forms an indispensable part of management of occupational health, which requires the occupational checkup physicians to shift their service capabilities and levels to meet more occupational groups' hope and demands on health.

\section{Implementation of Dynamic Strategies in Occupational Health}

Nowadays, more and more occupational groups are changing their recognition on health checkup, especially that of the chronic diseases caused by the lifestyles. They have stronger expectations to search checkup items which meet their own needs and really personalized and they are more willing to have dynamic management of occupational health.Therefore, how to implement dynamic management of occupational health becomes a problem faced by checkup institutions, employers, and occupational groups together.

As long as the checkup institutions reach an agreement with employers and occupational groups in terms of checkup items, the occupational groups' satisfaction and sense of acquisition will be ascended. However, the specified checkup items in the Technical Specification for Occupational Health Monitoring GBZ188-2014 ignored the status of occupational groups with different physical fitness and health. Hence, there author believes that the awareness of common management on occupational health between occupational checkup institutions and employers must be enhanced, with the requirements on knowing and agreement and corresponding articles of responsibilities made. The detailed implementation must specify the applicable and inapplicable groups to increase proper checkups from the perspective of regulations.

Many checkup institutions adopt occupational checkup software to enhance occupational groups' joy during checkup as there are more and more occupational groups year after year. However, their core interests lay in the maximization of profits from promoting the occupational groups' consumption. Therefore, only if the checkup institutions implement the 


ISSN 2664-0333 eISSN 2664-0341 http://dx.doi.org/10.6913/MRHK.202003_2(1).0006
conception of dynamic management of occupational health before, during, and after the checkups, the occupational groups' health rights and rights can be ensured.

The following aspects of efforts can be made to implement the dynamic management of occupational health. First, a linkage module to communicate with employers must be designed for their online contact, avoiding the tiny problems to affect the dynamic management of occupational health. Second, a signing service mode must be made jointly with the employers. The health education with expertise in occupational health and prevention and treatment of chronic diseases must be sent to the employers and occupational groups online randomly, to reduce the confusion caused by occupational groups' lack of medicine expertise and individual gaps of recognition, which enhances the conception of dynamic management of health while adopting health education at the same time. Third, physicians must take the initiative to consultation on the benefits of occupational health management face to face on the site of the employers.

In conclusion, implementation of dynamic management of occupational health checkup forms an important part to ensure the health right and interests of occupational groups. Nowadays, the industry involving checkup and health management is thriving day after day. It is worth our thinking whether we find our shortages and defects or not as we pursue benefits and whether the occupational checkup can meet the hope and demands of occupational groups or not. Here, it is hoped to make concrete improvement in the service mode of occupational health examination and concepts of services, realizing the target of "occupational health first" and striving to the "Healthy China" advocated by the nation accurately.

\section{REFERENCES}

[1]Zeng Qiang. How to Write a Good Chief Physician's Report. 2018 National Conference on Quality Control of Physical Examination. March, 2018.

[2]Xu Zhijie. Common Decision Must Be Advocated in Health Examination [J]. Chinese Journal of Health Management. April, 2018. 13.

[3]Li Shuang. The Framework of Thoughts and Contents to Construct a Health Enterprise. The 15th Symposium on the Prevention and Control of Occupational Diseases in the Four Provinces of Central China in 2018. Jun, 2018. 